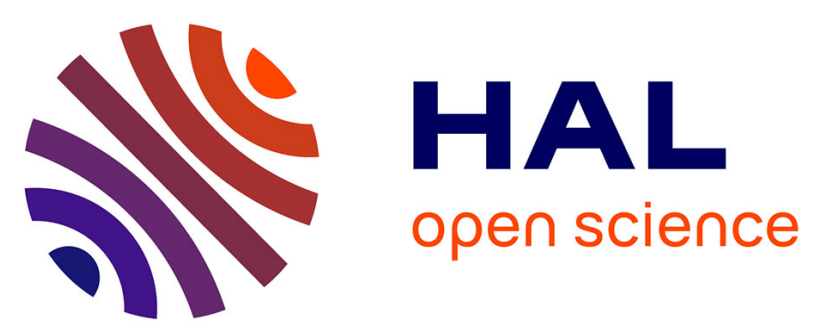

\title{
Calcul couplé fluide/structure avec Ansys : Etude numérique et expérimentale d'une plaque élastique en contact avec un fluide lourd compressible
} Jean-François Sigrist, Christian Lainé, Bernard Peseux

\section{- To cite this version:}

Jean-François Sigrist, Christian Lainé, Bernard Peseux. Calcul couplé fluide/structure avec Ansys: Etude numérique et expérimentale d'une plaque élastique en contact avec un fluide lourd compressible. Journal de Physique IV Proceedings, 2002, 12 (11), pp.137-144. 10.1051/jp4:20020486 . hal01915774

\author{
HAL Id: hal-01915774 \\ https://hal.science/hal-01915774
}

Submitted on 8 Nov 2018

HAL is a multi-disciplinary open access archive for the deposit and dissemination of scientific research documents, whether they are published or not. The documents may come from teaching and research institutions in France or abroad, or from public or private research centers.
L'archive ouverte pluridisciplinaire HAL, est destinée au dépôt et à la diffusion de documents scientifiques de niveau recherche, publiés ou non, émanant des établissements d'enseignement et de recherche français ou étrangers, des laboratoires publics ou privés. 


\title{
Calcul couple fluide/structure avec ANSYS : étude numérique et expérimentale d'une plaque élastique en contact avec un fluide lourd compressible
}

\author{
J.F. Sigrist, C. Laine et B. Peseux ${ }^{1}$ \\ Service Scientifique et Technique, Calcul et Simulation Numérique, DCN, \\ Établissement de Nantes-Indret, 44620 La Montagne, France \\ ${ }^{1}$ Laboratoire Mécanique et Matériaux, École Centrale de Nantes, 1 rue de la Noë, \\ 44321 Nantes cedex 3, France
}

\begin{abstract}
Résumé. Une préoccupation constante de tout industriel est la maîtrise des marges de dimensionnement de ses structures. Le recours aux techniques de calcul scientifique permet en phase de conception détaillée un dimensionnement au plus juste des structures, tout en respectant les différentes contraintes de conception (exigences fonctionnelles, conditions d'environnement, aspects réglementaires).

Une meilleure maîtrise des marges passe donc par une meilleure connaissance de l'outil de calcul (précision intrinsèque, mise en euvre de techniques de calcul plus ou moins élaborées) et la prise en compte d'une réalité physique plus complexe (avec par exemple un couplage entre différents phénomènes physiques) pour une meilleure représentativité du calcul. Une démarche globale de recherche et développement a été mise en place au sein du Service Scientifique et Technique de l'établissement DCN de Nantes Indret pour répondre à ce besoin. Nous présentons ici un exemple d'application pour le calcul de structures en présence de fluide. Cette étude numérique el expérimentale nous permet de valider conjointement le processus de calcul en bureau d'étude et les méthodes de mesure sur site dans le domaine de l'analyse fréquentielle de structures mouillées.
\end{abstract}

\section{LA MAITRISE DES MARGES DE DIMENSIONNEMENT}

La maîtrise des marges de dimensionnement est un enjeu à la fois technique et économique important pour tout industriel. Dans le processus industriel, une connaissance affinée des marges permet des gains économiques substantiels au moment de la conception détaillée et en situation de maintien en condition opérationnelle :

- en phase de conception détaillée, une bonne maîtrise des marges permet un dimensionnement au plus juste des structures et le gain généré dans ce cas par rapport à une structure surdimensionnée peut être important ;

- en phase de maintien en condition opérationnelle, une connaissance précise des marges permet de situer l'intervalle dans lequel il est pertinent de travailler et de privilégier une solution industrielle respectant à la fois les contraintes techniques et économiques pour la modification envisagée.

Un des moyens de maîtriser et d'améliorer ces marges de dimensionnement est d'accroître la connaissance de la précision des outils de calculs utilisés, de prendre en compte un ensemble de phénomènes physiques de plus en plus proche de la réalité opérationnelle et de travailler sur des critères de dimensionnement traduisant de façon la plus pertinente possible les exigences de résistance des structures aux sollicitations fonctionnelles et à l'environnement.

Une démarche de $R \& D$ a été mise en place au sein du Service Scientifique et Technique de l'établissement DCN de Nantes-Indret pour affiner la connaissance des marges de dimensionnement. Le principe de cette démarche est résumé dans la FIG. 1 ci-dessous. 
L'objet de l'exposé est de donner un exemple de réalisation de la première étape de cette démarche dans le cadre général du dimensionnement de structures en présence de fluide. L'étude du comportement dynamique de structures avec prise en compte du fluide a fait l'objet de nombreuses études [10] mais l'utilisation courante en bureau d'étude des techniques de calcul développées dans le cadre de ces recherches est suffisamment rare pour qu'on s'y intéresse de façon approfondie avant de mettre en place une méthode de calcul systématique appliquée à des structures industrielles.

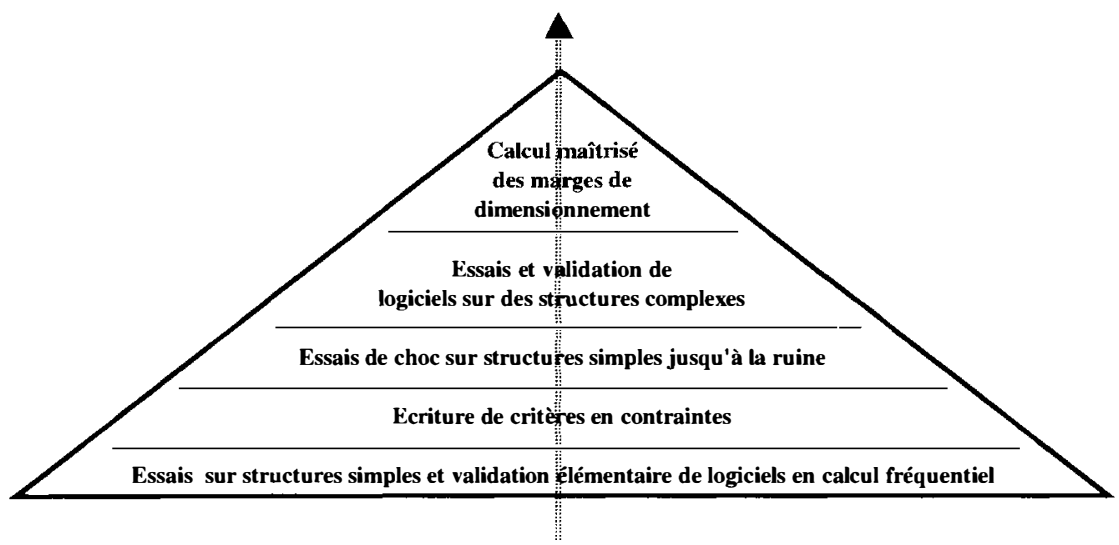

FIG. 1. DEMARCHE GENERALE R\&D DCN : VERS UN CALCUL MAITRISE DES MARGES DE DIMENSIONNEMENT

La mise en œuvre d'un calcul couplé fluide/structure nous conduit à nous intéresser à la modélisation théorique de ce type de problème, aux méthodes de résolution numérique utilisées et à l'application effective de ces techniques avec un code de calcul de structure généraliste. La connaissance des marges de dimensionnement passe ensuite par l'étude et l'explication des écarts constatés entre des résultats de calcul et des résultats expérimentaux de référence, réalisés en environnement maîtrisé.

\section{APPROCHE THEORIQUE ELEMENTAIRE DU COUPLAGE FLUIDE STRUCTURE. RESOLUTION NUMERIQUE PAR COUPLAGE ELEMENTS FINIS/ELEMENTS FINIS}

Le domaine d'étude est celui du comportement vibro-acoustique de structures en présence de fluide. La structure étudiée est supposée élastique, linéaire, homogène, isotope en petites transformations. Le fluide étudié est supposé parfait, newtonien, en petites transformations et pour lequel on néglige les effets de la pesanteur. La FIG. 2 ci dessous schématise le problème couplé, et précise les conventions de normales utilisées. Sous ces hypothèses, les équations descriptives du comportement de la structure et du fluide sont les suivantes.

L'équation locale dans le solide est [6] :

$$
\operatorname{div}\left(\overrightarrow{\vec{\sigma}}_{S}\right)+\vec{f}=\rho_{S} \frac{\partial^{2} \vec{u}_{S}}{\partial t^{2}}, \forall M \in \Omega_{S}
$$

Les conditions aux limites du domaine solide sont de type Dirichlet-Neumann :

$$
\vec{u}_{S}=\vec{u}_{d}, \forall M \in \delta \Omega_{S u} \text { et } \overrightarrow{\vec{\sigma}}_{S} \cdot \vec{n}_{S}=\vec{T}_{d}, \forall M \in \delta \Omega_{S \sigma}
$$

La condition de couplage avec le fluide exprime la continuité de la composante normale du tenseur des contraintes à l'interface fluide structure [10] et s'écrit :

L'équation locale dans le fluide est [3] :

$$
\overrightarrow{\bar{\sigma}}_{S} \cdot \vec{n}_{S}=p \vec{n}, \forall M \in \Gamma_{S F}
$$

$$
\Delta p-\frac{1}{c^{2}} \frac{\partial^{2} p}{\partial t^{2}}=0, \forall M \in \Omega_{F}
$$


Les conditions aux limites du domaine fluide sont de type Dirichlet-Neumann :

$$
p=p_{d}, \forall M \in \delta \Omega_{F u} \text { et } \frac{\partial p}{\partial n_{F}}=\left(\frac{\partial p}{\partial n_{F}}\right)_{d}, \forall M \in \delta \Omega_{F s}
$$

La condition de couplage avec le solide traduit continuité de la composante normale de l'accélération à l'interface fluide structure [10] et s'écrit :

$$
\frac{\partial P}{\partial n}=-\rho_{F} \frac{\partial^{2} \vec{u}_{S}}{\partial t^{2}} \cdot \vec{n}, \forall M \in \Gamma_{F S}
$$

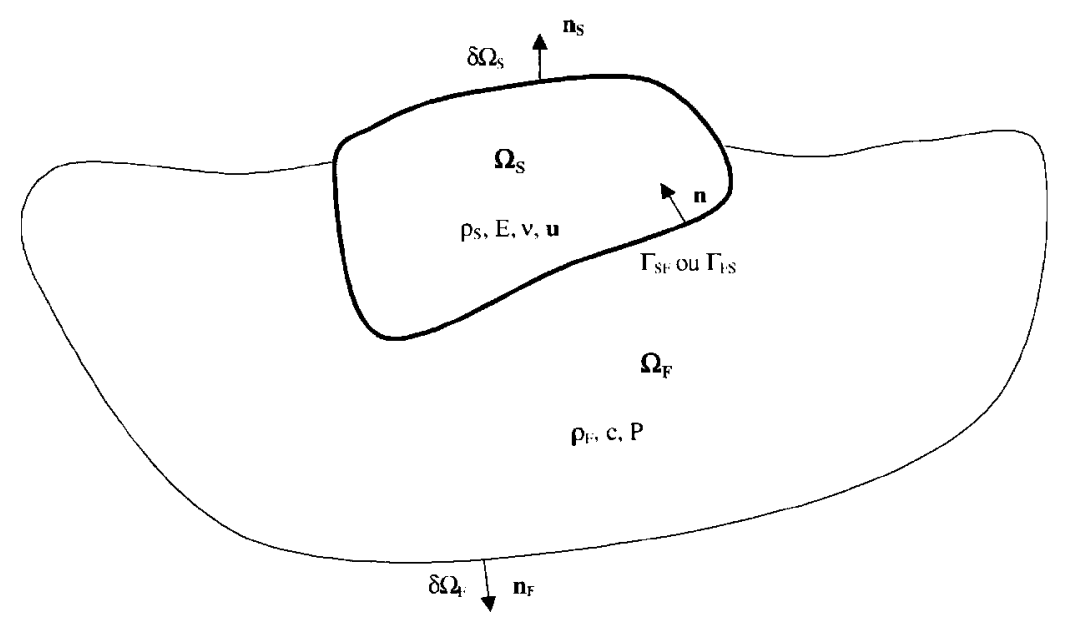

FIG. 2. SCHEMATISATION D'UN PROBLEME COUPLE FLUIDE/STRUCTURE

La méthode de résolution numérique du problème aux valeurs propres ainsi posé se fait sous ANSYS par couplage entre une discrétisation de type éléments finis du problème fluide (formulation en déplacement) et du problème structure (formulation en pression) [13]. On s'intéresse aux fréquences propres du système couplé : après transformée de Fourrier des équations précédentes, on écrit la formulation variationnelle correspondante [4], en notant $\delta \bar{u}_{S}$ le déplacement virtuel et $\delta p$ la variation virtuelle de pression :

$$
\begin{aligned}
& \int_{\Omega_{S}} \overrightarrow{\vec{\sigma}}_{S}\left(\vec{u}_{S}\right) \cdot \overrightarrow{\vec{\varepsilon}}_{S}\left(\delta \vec{u}_{S}\right) d \Omega-\omega^{2} \int_{\Omega_{S}} \rho_{S} \vec{u}_{S} \cdot \delta \vec{u}_{S} d \Omega-\int_{\Gamma_{S t}} p \vec{n} \cdot \delta \vec{u}_{S} d \Gamma=0 \\
& \int_{\Omega_{F}} \nabla p \cdot \nabla \delta p d \Omega-\frac{\omega^{2}}{c^{2}} \int_{\Omega_{t}} p \delta p d \Omega-\omega^{2} \int_{\Gamma_{r S}} \rho_{F} \vec{u}_{S} \cdot \vec{n} \delta p d \Gamma=0
\end{aligned}
$$

Une discrétisation de type éléments finis [16] avec la méthode de Galerkin, appliquée à ces deux équations conduit au système matriciel suivant :

$$
\left(\left[\begin{array}{cc}
K_{S} & -R_{F S} \\
0 & K_{F}
\end{array}\right]-\omega^{2}\left[\begin{array}{cc}
M_{S} & 0 \\
\rho_{F} R_{S F} & M_{F}
\end{array}\right]\right)\left\{\begin{array}{c}
U_{S} \\
P
\end{array}\right\}=\left\{\begin{array}{l}
0 \\
0
\end{array}\right\}
$$

La formulation déplacement/pression génère un problème aux valeurs propres généralisé non symétrique du fait de la présence des termes de couplage exprimés en fonction de la matrice d'interaction fluide/structure, calculée à l'interface $\Gamma_{\mathrm{SF}}$ à partir des fonctions d'interpolation fluide et structure et de la normale locale :

$$
\left[R_{F S}\right]=\left[R_{S F}\right]^{T}=\int_{\Gamma_{S F}}\left[N_{S}\right]^{T}\{n\}<N_{F}>d \Gamma
$$

D'autres formulations sont possibles et permettent de travailler avec un problème complètement symétrique [7], en particulier des formulations de type déplacement/déplacement [2]. De telles 
formulations sont disponibles avec ANSYS [8], mais leur mise en œuvre peu aisée dans le cas de géométries complexes les rendent difficilement utilisables en bureau d'étude pour traiter des cas industriels.

Selon le type de conditions aux limites, le problème aux valeurs propres concerne les matrices complètes ou des sous matrices; on ne conserve que les lignes et colonnes correspondant à des nœuds non contraints [9]. Deux types de conditions aux limites sur le domaine fluide sont possibles [15] :

- condition de pression nulle : cette condition représente soit une surface libre du fluide $(p=0)$, soit un plan d'anti-symétrie du domaine fluide ;

- condition de pression libre : cette condition représente soit la présence d'une paroi rigide en contact avec le fluide $\left(\frac{\partial p}{\partial n}=0\right)$, soit un plan de symétrie du domaine fluide.

La résolution du problème aux valeurs propres généralisé non symétrique avec ANSYS utilise l'algorithme de Lanczos non symétrique [12]. Une application directe de cette méthode de résolution est proposée dans le paragraphe suivant. On réalise une étude paramétrique destinée à évaluer l'influence des différentes hypothèses de modélisation sur le résultat du calcul.

\section{MISE EN GUVRE D'UN CALCUL COUPLE. ETUDE PARAMETRIQUE}

Le problème étudié est celui d'une plaque encastrée en ses bords, plongée dans un fluide compressible non borné. Les caractéristiques du fluide sont celle de l'eau à $20^{\circ} \mathrm{C}$, les caractéristiques de la plaque sont celles de l'acier. La modélisation ANSYS utilise des éléments solid45 : éléments linéaires à huit nœuds, trois degrés de liberté par nœud (composantes $u, v$ et $w$ du déplacement) et des éléments fluid30: éléments linéaires à huit nœuds, un degré de liberté par nœud (pression $p$ ).

Un calcul de référence est réalisé à partir d'un maillage standard. Ce résultat est ensuite comparé à un calcul pour lequel on fait varier un paramètre donné. L'ensemble des expérimentations numériques couvertes est proposé de manière exhaustive dans [14]. L'analyse des différents résultats nous permet d'apprécier l'influence des différents éléments suivants sur la précision du calcul :

- taille du domaine fluide modélisant un espace non borné (dimensions spatiales);

- qualité de maillage (nombre, taille et élancement des éléments) ;

- caractéristiques du fluide (célérité des ondes acoustiques, masse volumique) ;

- conditions de couplage (déclaration des interfaces) ;

- conditions aux limites (pression nulle ou pression libre);

- exploitation des symétries (conditions de symétrie ou d'anti-symétrie).

A l'issue de cette étude, nous sommes capables de produire un maillage dont les caractéristiques sont ajustées pour nous permettre de modéliser une plaque élastique plongée dans un fluide acoustique. Les observations faites lors de l'étude paramétrique permettent, compte tenu de la linéarité du problème, d'établir des règles de maillage applicables à des problèmes dont la géométrie est plus complexe.

Nous souhaitons maintenant confronter des résultats de calculs relatifs à des plaques couplées avec un fluide dans des situations où l'on dispose de solutions analytiques, de résultats d'essais en laboratoire et de calculs numériques associés.

\section{CONFRONTATION DU LOGICIEL ANSYS AVEC DES CALCULS DE REFERENCE}

\subsection{Plaque simplement appuyée en contact avec un fluide borné compressible avec conditions de surface libre}

Le problème est présenté de façon détaillée dans [5]. Dans ce cas d'étude, on dispose d'une solution analytique. Si la plaque est de dimensions $a \times b \times e$, de caractéristiques $E, \rho_{S}, v$, si $h$ est la hauteur de 
fluide, de caractéristiques $c$ et $\rho_{F}$, alors les pulsations propres du système couplé sont les racines de l'équation suivante :

$$
\frac{E e^{3}}{12}\left(1-v^{2}\right)\left[(n \pi / a)^{2}+(m \pi / b)^{2}\right]^{2}-\rho_{S} a b e \omega^{2}-\rho_{F} \frac{\tan (k h)}{k}=0
$$

avec $k=\sqrt{\omega^{2} / c^{2}-\left[(n \pi / a)^{2}+(m \pi / b)^{2}\right]^{2}}$ et $(n, m) \in \mathbf{N}^{2}$. Les pulsations propres dépendent donc du mode de déformation de la plaque (défini par les indices $n$ et $m$ ) et de la hauteur $h$ de fluide présent audessus de la plaque.

La FIG. 3 suivante donne pour trois modes une comparaison entre les valeurs des pulsations des modes évanescents calculés par ANSYS et les résultats théoriques attendus, en fonction de la hauteur de fluide. L'ensemble des résultats est présenté dans [14]. Les écarts relevés sont inférieurs à $1.5 \%$ pour toutes les hauteurs de fluide considérées et pour tous les modes étudiés. La corrélation ANSYS/théorie est donc excellente dans ce cas d'étude.

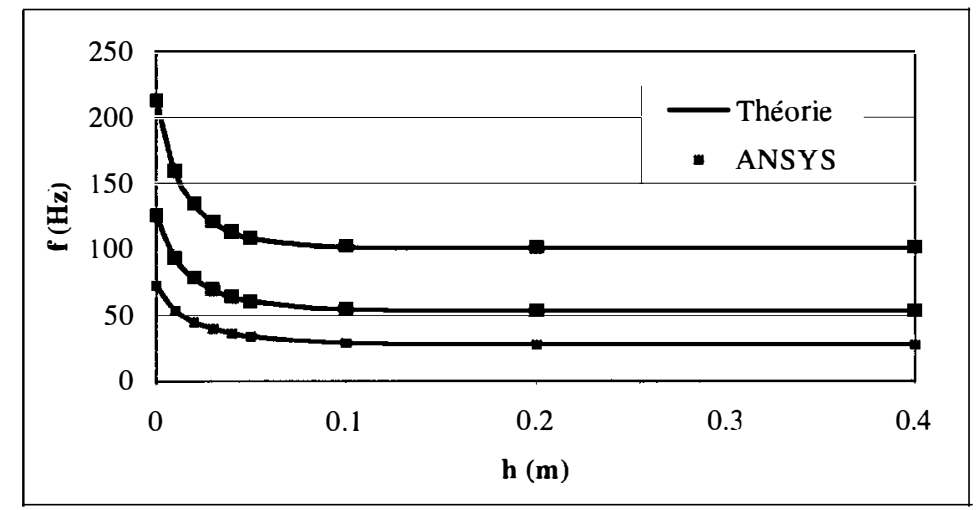

FIG. 3. COMPARAISON DES RESUlTATS DE CALCUL ANSYS AVEC LA SOLUTION THEORIQUE

\subsection{Plaque libre/libre immergée dans un fluide non borné compressible}

Le problème étudié est présenté dans [11]. On dispose pour ce cas d'étude de résultats d'essais de laboratoire, corrélés avec des résultats de calcul utilisant des méthodes de résolution numérique différentes (discrétisation du problème fluide par éléments finis, éléments finis et éléments infinis, équations intégrales). Le TAB. 1 ci-dessous propose une comparaison entre les résultats d'essais, les résultats de calcul numérique de référence et les résultats de calcul ANSYS.

On note une très bonne corrélation entre les résultats de calcul ANSYS et les résultats numériques de référence (l'écart moyen constaté est de l'ordre de 1.0\%); on constate également une très bonne corrélation entre les résultats de calcul ANSYS et les résultats expérimentaux (écart moyen constaté de l'ordre de 3\%).

TAB. 1. PlaQue PARTIELlEMENT IMMERGEE DANS UN FLUIDE LOURD. COMPARAISON ENTRE LES RESUltATS DE CALCUL ANSYS, DES RESULTATS EXPERIMENTAUX ET DES RESULTATS DE CALCUL NUMERIQUES DE REFERENCE.

\begin{tabular}{|lccccccc|}
\hline & $\mathbf{f}_{\mathbf{1}}$ & $\mathbf{f}_{\mathbf{2}}$ & $\mathbf{f}_{\mathbf{3}}$ & $\mathbf{f}_{\mathbf{4}}$ & $\mathbf{f}_{\mathbf{5}}$ & $\mathbf{f}_{\mathbf{6}}$ & $\mathbf{f}_{\mathbf{7}}$ \\
Exp. & 160.0 & 258.9 & 435.7 & 565.8 & 897.2 & 923.6 & 1144.5 \\
Réf. & 160.6 & 268.0 & 440.2 & 591.1 & 915.2 & 968.2 & 1172.8 \\
ANSYS & 162.9 & 270.3 & 445.4 & 592.2 & 920.2 & 967.7 & 1138.3 \\
$\varepsilon_{\text {ANSYS/exp. }}$ & $1.81 \%$ & $4.40 \%$ & $2.22 \%$ & $4.66 \%$ & $2.56 \%$ & $4.77 \%$ & $-0.54 \%$ \\
$\varepsilon_{\text {ANSYS/réf. }}$ & $1.43 \%$ & $0.86 \%$ & $1.18 \%$ & $0.18 \%$ & $0.55 \%$ & $-0.05 \%$ & $-2.94 \%$ \\
\hline
\end{tabular}


D'autres comparaisons sont proposées dans [14]. Nous avons mis en évidence un très bon comportement d'ANSYS dans la modélisation d'une plaque en contact avec un fluide. Les comparaisons présentées montrent donc que les performances de l'outil de calcul ANSYS sont dans ce domaine comparables avec les performances des outils de calcul développés dans le cadre de recherches universitaires.

\section{ESSAIS DE VIBRATION DE STRUCTURES SIMPLES EN ENVIRONNEMENT NON MAITRISE}

L'étude présentée au paragraphe suivant nous a permis d'évaluer les écarts entre un calcul ANSYS et des résultats d'essais réalisés dans des conditions idéalisées et maîtrisées. Le recalage d'un modèle numérique élaboré en bureau d'étude se fait à partir de données d'essais réalisés en conditions opérationnelles : à la différence des expérimentations en laboratoire, les manipulations mises en place dans un contexte industriel sont effectuées dans un environnement non maitrisé.

Il est intéressant de vérifier que les conditions d'essais ne perturbent pas le résultat de mesure et que l'écart entre un résultat d'expérience en environnement non maîtrisé est du même ordre de grandeur que celui mis en évidence par les comparaisons proposées dans le paragraphe précédent.

Dans ce but, nous avons mis en place une campagne d'essais de deux jours sur un site DCN. Après avoir mesuré les premières fréquences propres de structures simples (plaque mince, plaque épaisse, cylindre court, cylindre élancé) en air, celles-ci ont été placées en immersion totale en mer : un plongeur était chargé des excitations au marteau de choc étanche et l'acquisition des mesures était réalisée depuis une embarcation d'essais. On a cherché une profondeur de travail suffisamment importante pour placer les structures dans des conditions de fluide non borné, tout en respectant les contraintes de la manipulation (profondeur de travail du plongeur, longueur des câbles de mesure, etc).

La FIG. 4 suivante visualise les conditions de réalisation de ces essais de vibration. Les différents paramètres d'environnement - tels que les caractéristiques physiques et les mouvements du fluide, les conditions aux limites fluide et solide, la qualité de la manipulation en eau, etc - ne sont pas totalement maitrisés par l'expérimentateur.
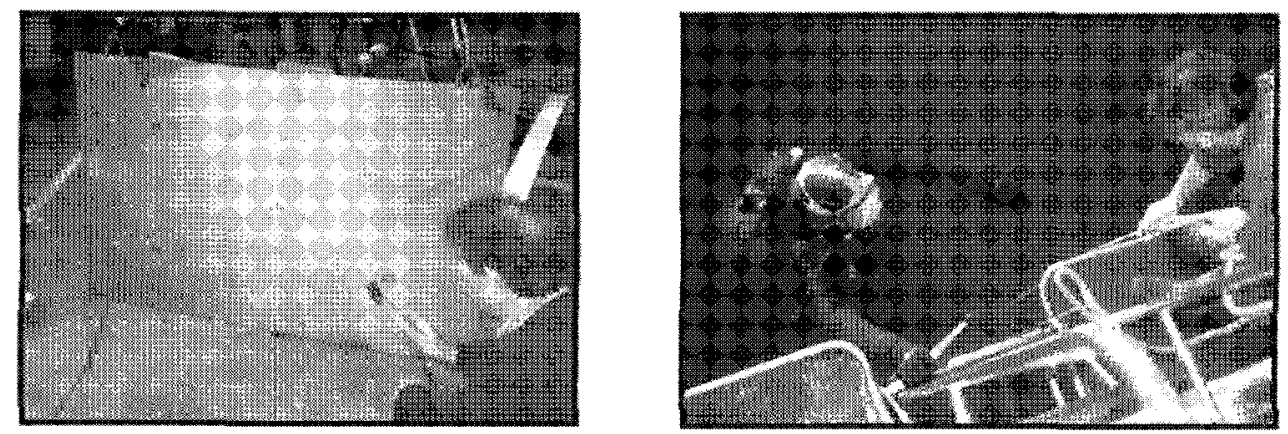

Fig. 4. CONDITIONS DE REALISATION D'ESSAIS DE VIBRATION DE STRUCTURES SIMPIES (EN AIR ET EN EAU) EN ENVIRONNEMENT NON MAITRISE

A partir des constatations numériques faites lors des études paramétriques et numériques précédentes, nous élaborons un maillage du problème physique pour lequel les erreurs de calculs inhérentes aux hypothèses de modélisation (caractéristiques du milieu fluide, conditions aux limites appliquées, qualité et forme du maillage) sont identifiées et maîtrisées. L'exploitation des symétries permet à précision égale de gagner en temps de calcul, en réduisant le problème complet ( 22.000 degrés de liberté) à un huitième du problème (3.300 degrés de liberté) avec des conditions de symétrie respectant les déformées modales. 
Le TAB. 2 ci-dessous donne les écarts constatés entre le résultat de calcul ANSYS et les mesures effectuées sur la plaque mince.

TAB. 2. ECARTS RELEVES ENTRE LES RESULTATS DE CALCUL ANSYS ET LES RESULTATS EXPERIMENTAUX

\begin{tabular}{|ccccccccc|}
\hline Fréquence & $\mathbf{f}_{\mathbf{1}}$ & $\mathbf{f}_{\mathbf{2}}$ & $\mathbf{f}_{\mathbf{3}}$ & $\mathbf{f}_{\mathbf{4}}$ & $\mathbf{f}_{\mathbf{5}}$ & $\mathbf{f}_{\mathbf{6}}$ & $\mathbf{f}_{\mathbf{7}}$ & $\mathbf{f}_{\mathbf{8}}$ \\
Ecart en AIR & $1.0 \%$ & $4.3 \%$ & $0.8 \%$ & - & $-1.9 \%$ & $-0.5 \%$ & $5.7 \%$ & $-3.0 \%$ \\
Ecart en EAU & $0.5 \%$ & - & $-2.2 \%$ & $-7.7 \%$ & $5.4 \%$ & $-5.4 \%$ & $-6.4 \%$ & $-3.5 \%$ \\
\hline
\end{tabular}

Les mesures en air ne permettent pas d'obtenir le mode $n^{\circ} 4$. En fait, les modes $n^{\circ} 3$ et $\mathrm{n}^{\circ} 4$ calculés se situent à des niveaux de fréquences trop proches pour être distingués par nos mesures compte tenu de la sensibilité de l'appareil utilisé. Les mesures en eau ne nous ont pas permis de mettre en évidence le deuxième mode de vibration de la plaque couplée. Dans la gamme de fréquence concernée, la qualité médiocre des mesures effectuées explique cette observation.

L'examen des résultats de calcul montre une très bonne corrélation avec les mesures réalisées. Les erreurs commises sont du même ordre de grandeur que celles constatées lors des comparaisons avec des essais de laboratoire. Le mode opératoire mis en œuvre dans nos essais, qui est représentatif du protocole expérimental utilisé par DCN pour la mesure in situ des fréquences propres d'un élément du navire (par exemple les safrans), n'est donc pas de nature à modifier sensiblement les résultats de mesure.

L'étude ainsi réalisée a permis de valider conjointement le processus de calcul et le mode opératoire des mesures pour l'étude fréquentielle de structures mouillées.

\section{CONCLUSION}

L'objectif de la présente étude était de mieux connaitre les marges de dimensionnement de structures en présence de fluide. Une étude bibliographique succincte réalisée autour des problèmes de modélisation physique de systèmes couplés et de résolution numérique des équations mathématiques ainsi posées nous a permis de mieux connaître les aspects théoriques relatifs à la modélisation de problèmes couplés fluide/structure. Nos recherches bibliographiques nous ont conduit à étudier un certain nombre de cas de validation de modèles et d'outils de calcul par confrontation avec des résultats d'essais réalisés en laboratoire.

L'essentiel des études de recherche se situe entre ces deux ensembles : aspects théoriques d'une part et expérimentations en milieu maîtrisé d'autre part, une confrontation entre les deux permettant de valider les méthodes numériques et les outils utilisés. Nous avons appliqué cette démarche à l'outil de calcul ANSYS ce qui nous a permis de nous approprier le code et de maîtriser l'ensemble de la chaîne de calcul.

Nous avons ensuite choisi de sortir d'un cadre idéalisé pour confronter notre démarche de calcul avec la réalité opérationnelle telle qu'elle se présente à l'industriel. Des essais sur structures simples en environnement non maîtrisé ont été réalisés et nous avons confronté ces résultats d'essais avec les calculs effectués selon le mode opératoire préalablement validé.

Après analyse, il est montré que :

- les mesures réalisées en conditions opérationnelles donnent des résultats satisfaisants ;

- l'origine des écarts entre la réalité opérationnelle et les résultats de calcul menés en bureau d'étude est mieux expliquée ;

- la connaissance des marges de dimensionnement en est donc améliorée. 
Le processus de calcul étant ainsi validé dans tous ces aspects, on peut envisager son application en bureau d'étude : une étude industrielle en cours doit permettre la justification de la tenue au séisme d'un prototype à terre d'un système d'énergie propulsion, avec prise en compte de l'interaction fluide structure.

Les limites d'une approche linéaire ne permettent cependant pas de couvrir tous les cas de fonctionnement des systèmes d'énergie propulsion auxquelles l'établissement DCN de Nantes-Indret s'intéresse. En particulier, certains cas de fonctionnement de ces systèmes nécessitent la prise en compte des mouvements du fluide, en général sous pression, ainsi que de grands déplacements de la structure à dimensionner. Ces points feront l'objet de développements ultérieurs pour lesquels on envisage de modéliser l'interaction fluide structure en réalisant un couplage entre un code de mécanique des fluides (discrétisation de type volumes finis) avec un code de mécanique des structures (discrétisation de type éléments finis).

\section{REFERENCES BIBLIOGRAPHIQUES}

[1] R.D. BLEvins. Formulas for Frequence and Mode Shape. Robert E. Kreieger Publishing Compagny, 1984.

[2] J. Boujot. Mathematical Formulation of Fluid Structure Interaction Problems. Mathematical Modelling and Numerical Analysis, 21, 239-260, 1987.

[3] S. CANDEL. Mécanique des fluides. Dunod, 1995.

[4] P.G. CIARLET. Introduction à l'analyse numérique matricielle et à l'optimisation. Masson, 1988.

[5] B. Costa et al. Analyse vibro-acoustique d'une plaque couplée avec un fluide lourd. $J$. Acoustique, 5, 543-554, 1992.

[6] G. Duvaut. Mécanique des milieux continus. Masson, 1990.

[7] G. C. Everstine. Letter to the Editor: A symettric Potential Formulation fort FluidStructure Interaction. Journal of Sound and Vibration, 79 (1), 157-190, 1981.

[8] P. KHONKE. ANSYS Theory Reference. 5.5. Release. Swanson Analysis System Inc, 1986.

[9] M.S. MarcuS. A Finite Element Method Applied to the Vibration of Submersed Plates. $J$. Ship Research, 22 (2), 94-99, 1978.

[10] H. J.P. MORAND and R. OHAYON. Fluid Structure Interaction. Wiley \& Sons, 1995.

[11] B. PESEUX et J.P. QUEVAT. Vibration d'une plaque immergée dans un fluide incompressible. Groupe Analyse Dynamique. Validation des progiciels de calcul de structures. SFM, 1990.

[12] C. Rajakumar and C.G. Rogers. The Lanczos Algorithm Applied to Unsymmetric Generalized Eignevalue Problem. Int. J. Num. Methods in Engineering, 32, 1009-1026, 1991.

[13] E.A. SCHROEDER and M.S. MARCUS. Finite Element Solution of Fluid-Structure Interaction Problems. $46^{\text {th }}$ Shock and Vibration Symposium. San Diego, October 1975.

[14] J.F. Sigrist. Analyse numérique détaillée d'une plaque élastique en contact avec un fluide compressible. Confrontations aux essais expérimentaux. Mémoire de DEA Génie Mécanique, Calcul de Structures. Ecole Centrale de Nantes, 2001.

[15] D.B. WoYAK. Acoustic and Fluid Structure Interaction. Revision 5.0 Tutorial. Swanson Analysis System Inc, 1995.

[16] O.C. ZIENKIEWICZ and R.L. TAYLOR. The Finite Element Method. Basic Formulation and Linear Problems. Mac Graw Hill, 1989. 